Letters to the Editor

\section{Correlation Between Healthcare Workers' Knowledge of Influenza Vaccine and Vaccine Receipt}

\section{To the Editor:}

A recent study published in Infection Control and Hospital Epidemiology evaluated the correlation between healthcare workers' knowledge about influenza vaccine and acceptance of vaccination. ${ }^{1}$ Professionals with greater knowledge about the usefulness of the vaccine, as demonstrated by correctly answering 5 questions, had higher vaccination rates than did those answering 1 or more of the questions incorrectly. Our research group performed a similar study using a questionnaire administered to 551 healthcare workers in primary healthcare centers in Catalonia, Spain, and found similar results. We also used a Likert scale of 0 (vaccination completely useless) to 5 (vaccination very useful) to establish how the vaccine was perceived by healthcare professionals.

As in the study by Martinello et al., those who believed that the vaccine worked were more likely to be vaccinated themselves (4.2 on the Likert scale vs 3.3 for those not vaccinated). Primary care physicians were the group who considered the vaccine most useful (average score, 3.77), followed by pediatricians (3.63). Significantly lower scores were recorded for nurses $(3.44 ; P<$ $.01)$.

\section{REFERENCE}

1. Martinello RA, Jones L, Topal JE. Correlation between healthcare workers' knowledge of influenza vaccine and vaccine receipt. Infect Control Hosp Epidemiol 2003;24:845-847.

Carl Llor, PhD

Luisa Morató, MD

Purificación Robles, MD

Ariadna Mas, MD

Catalan Institute of Health

Tarragona, Spain

The authors decline to reply.

\section{An Estimate of the Incidence of Candiduria Among Hospitalized Patients in the United States}

\section{To the Editor:}

Candida species are commonly isolated from the urine cultures of hospitalized patients. ${ }^{1}$ Candida urinary isolates are second only to Escherichia coli in combined medical-surgical intensive care units (ICUs) ${ }^{2}$ Because indications for treating it are often unclear, candiduria presents as a therapeutic dilemma to clinicians, who frequently treat with antifungal agents despite not knowing for certain whether Candida represents colonization or true infection. ${ }^{3}$ Whereas there is evidence that candiduria is becoming an increasing problem, little is known about its actual incidence. In this era of increasing attention toward developing costeffective methods to manage infectious diseases, ${ }^{4}$ it is important to determine the incidence of candiduria so that the impact of interventions to manage it can be ascertained.

We performed a systematic review of the literature using PubMed with the keywords "candid"," "urinary," "infection," and "incidence" to identify published data on the incidence of candiduria. Additionally, we obtained the rate of urinary tract infections (UTIs) per 1,000 urinary catheter-days and the rate of urinary catheter-days per ICU patient-days from National Nosocomial Infections Surveillance (NNIS) System data pertaining to UTIs. ${ }^{5}$ Because the NNIS System reports pooled mean UTI rates according to individual ICU type, we calculated the overall mean UTI rate by (1) multiplying the pooled mean UTI rate by the number of urinary catheter-days of each ICU type to obtain the number of UTIs, (2) calculating the sum of UTIs, and (3) dividing the sum of UTIs by the sum of urinary catheter-days. The overall mean rate of urinary catheter-days was calculated in a similar fashion. The number of ICU beds in the United States (excluding neonatal beds) was obtained from the annual hospital survey of the
American Hospital Association. ${ }^{6}$ We estimated the number of ICU-days in the United States by applying the mean occupancy rate of ICUs reported by Groeger et al. to the number of ICU beds and multiplying by 365.25 days. ${ }^{7}$ To calculate the number of nosocomial UTIs, we multiplied the number of ICU-days by the number of catheterdays per ICU-days and by the number of UTIs per 1,000 catheter-days. We then multiplied the number of nosocomial UTIs by the percentage of UTIs caused by Candida species to obtain the incidence of candiduria.

In the United States, there were an estimated 5.3 UTIs per 1,000 ICU catheter-days from January 1995 to June 2001. ${ }^{5}$ The rate of urinary catheter-days per ICU patient-days was 0.73 . According to the 2001 American Hospital Association survey, there were 69,593 ICU beds in the United States, with a mean occupancy rate of $84 \% .6$ Because there were approximately 21.3 million ICU-days per year during this period, there are an estimated 82,000 nosocomial UTIs per year in ICUs in the United States. Given that approximately $31 \%$ of all nosocomial UTIs are caused by Candida species, ${ }^{1}$ we estimate the incidence of candiduria in the intensive care setting to be approximately 25,000 cases per year in the United States.

We have demonstrated that candiduria is a common problem in ICUs in the United States. In our review of the literature, we found only one previous study specifically addressing the incidence of candiduria in the United States, but this study was limited to a single university hospital. ${ }^{8}$ Currently, there are no precise estimates of the incidence of UTIs because UTIs are not reportable diseases, and there are no large-scale surveillances of UTIs except in the ICU setting. However, treatment of candiduria is common. A multicenter U.S. study showed that $43 \%$ of Candida isolates in urine were treated, suggesting that candiduria is associated with significant antimicrobial use. ${ }^{9}$

Several factors limit the accuracy of our estimate of the incidence of candiduria. First, our estimate does not account for candiduria in non-ICU or neonatal ICU patients, because the NNIS System does not collect data from these settings. Second, there are currently limited data regarding non- 